\title{
Introduction to the eleventh Spondyloarthritis Research and Therapy Network (SPARTAN) annual research and education meeting
}

\author{
Luis R. Espinoza
}

Received: 31 January 2014 / Accepted: 24 March 2014 / Published online: 9 May 2014

(C) Clinical Rheumatology 2014

The Spondyloarthritis Research and Therapy Network (SPARTAN) is a network of health care professionals who are dedicated to research, awareness, and treatment of ankylosing spondylitis (AS) and related forms of spondyloarthritis (SpA). The group was founded in 2003 and is funded through educational grants from companies with an interest in SpA. SPARTAN members meet yearly to promote research, education, and treatment of SpA; proceedings from previous meetings have been reported elsewhere.

The SPARTAN 2013 meeting was held on August 2 to 3 in San Francisco, CA. A series of major presentations was given that illustrates recent advances on the pathophysiology, etiology, immunogenetics, classification criteria, axial and peripheral clinical manifestations, and also on the development on an American College of Rheumatology (ACR)-SPARTAN initiative on the development of newer criteria for its therapeutic management. Some of the major presentations will be summarized.

The opening article reviews the initiative to develop treatment guidelines for axial spondyloarthritis (SpA) including ankylosing spondylitis (AS) that began in 2011 as a collaborative effort between the ACR and SPARTAN, with support from the Spondylitis Association of America, a patient education and advocacy organization. The project will use the Grading of Recommendations Assessment, Development, and Evaluation (GRADE) method, which is based on systematic literature reviews and quantitative evidence summaries, to develop treatment recommendations for the use of pharmacological interventions, rehabilitation, surgery, preventive care, and disease monitoring in patients with AS and axial

L. R. Espinoza $(\bowtie)$

Louisiana State University Health Sciences Center, New Orleans, LA. 1542 Tulane Avenue, New Orleans, LA 70122-2822, USA

e-mail: lespin1@1suhsc.edu spondyloarthritis. The AS/axial SpA guidelines are anticipated to be developed and disseminated by late 2014 .

A critical appraisal of the Assessment of SpondyloArthritis International Society (ASAS) classification criteria for axial spondyloarthritis (axSpA) developed in 2009 and also of the modified New York ( $\mathrm{mNY}$ ) criteria for the classification of AS is discussed next. In the absence of "diagnostic" criteria for either axSpA or AS, both of these "classification" criteria are routinely used in clinical practice to diagnose patients. This issue raises serious concerns, especially "misdiagnosis" of individual patients if classification criteria are misapplied. The majority of SPARTAN members present at the meeting agree that SPARTAN should participate in either modifying the existing criteria or develop new diagnostic criteria for spondyloarthritis.

The next three articles review basic concepts of etiopathogenesis in the spondyloarthritides. An update on the contribution of the MHC to AS susceptibility is discussed first. The strong association of human leukocyte antigen (HLA)-B27 with AS is emphasized as one of the best examples of disease association with a hereditary marker, although genome-wide association and family studies support the presence of other important MHC influences in AS susceptibility. The mechanism(s) on how the presence of HLA-B27 is further explored, as well as other HLA class I and class II and other non-HLA allelic associations.

One of the most intriguing issues in the pathogenesis of AS and related disorders are the events that lead to bony fusion. This issue is discussed next in which the potential impact of the Wnt signaling pathway is explored. This pathway is involved in bone morphogenesis and homeostasis, and dysregulation has been implicated in abnormal bone formation. In addition, the role of levels of Wnt regulatory proteins like Dickkopf as potential biomarker of disease is also reviewed.

The final article is of special interest and discusses the potential role of the microbiome in the pathogenesis of 
spondyloarthritis. Evidence is presented that strongly implicates bacteria in the pathogenesis of spondyloarthritis. In addition, a brief overview on how HLA-B27 may influence the microbiome is presented. Of particular interest and complementing the discussion presented is the recently published observation that expression of HLA-B27 leads to a defect in interferon- $\gamma$ (IFN $\gamma$ ) signaling in antigen-presenting cells in both B27-transgenic rats and patients with SpA. In the case of B27-transgenic rats, the defect may cause the expansion of Th17 cells and alter regulatory $\mathrm{T}$ cell function, both of which can contribute to the pathogenesis of SpA [1].

\section{Reference}

1. Fert I, Cagnard N, Glatigny S et al (2014) Reverse interferon signature is characteristic of antigen-presenting cells in human and rat spondyloarthritis. Arthritis Rheumatol 66:841-862 